was considerably dilated. In six cases the murmur was in the first part of the diastole and in every one of these the left ventricle was found to be markedly dilated and usually also hypertrophied. So it seems that the early diastolic murmur is heard in those cases of mitral stenosis in which the left ventricle is dilated. Now, in mitral stenosis alone the left ventricle does not dilate; it may either become smaller, as it receives less blood, or it may become slightly hypertrophied, possibly to increase its suction power, as suggested by Giuffré. 2 If with mitral stenosis we find distinct left ventricular dilatation we may be sure, excluding other valvular or visceral disease, that during life there was considerable mitral regurgitation, in which condition dilatation is of course frequent. This great frequency of dilatation of the left ventricle in cases of mitral stenosis with early diastolic murmur, together with the clinical association of such murmurs with systolic bruits, and the fact that this murmur is only heard when compensation is failing, leads to the conclusion that the murmur in the first part of the diastole is only heard in cases of mitral stenosis in which there is free mitral regurgitation. The objection might be raised that with the rigid and useless valves of mitral stenosis there must be very frequently regurgitation and that the early diastolic murmur skould be therefore more common. But there are frequently no signs during life or after death that regurgitation has taken place and, as suggested by Samways, 3 this may be due to hypertropby of the left auricle which, until it gives way, prevents by its more forcible contraction any great mitral regurgitation. The rapid changes in the time of the murmur of stenosis may be due to alterations in tone of the muscle of the left auricle and auriculo-ventricular ring, allowing greater or less regurgitation.

Although there have been no suggestions as to the significance of the early diastolic as compared with the presystolic murmur, yet there are several explanations as to the mode in which it is produced. There is the view supported by Fagge ${ }^{4}$ that it is caused by an increased duration of the auricular systole, so that it drives the blood through the narrowed orifice as soon as the ventricle dilates. Apart from the difficulty of conceiving such a cardiac rhythm on physiological grounds there is the obvious objection that such murmurs are very frequently heard with, but separated from, presystolic murmurs which would be most unlikely if they were both due to the auricular contraction. Balfour ${ }^{5}$ ascribes this murmur to the high tension in the pulmonary system forcing blood through the narrow mitral orifice to fill the left ventricle as soon as it is emptied. If this were sufficient by itself to produce a bruit we should expect an early diastolic murmur in all advanced cases of mitral stenosis until the right side gives way, relieving the high pulmonary pressure, whereas such murmurs are only heard in a minority of cases. There is also the attractive theory put forward by Rolleston ${ }^{6}$ and adopted by Gibson ${ }^{7}$ and others that the early diastolic murmur is due to the negative pressure in the expanding left ventricle sucking blood through the constricted orifice. If the early diastolic murmur is associated with a dilated left ventricle, as appears from my analysis of post-mortem examinations, which unfortunately includes only a small number of cases, it can hardly be explained by this vis a fronte. For eren if we suppose the wall of the left ventricle to remain of the same thickness and elasticity when the cavity is dilated there would be as a question of physics less negative pressure within it; and as the wall of a dilated ventricle is always softer and less elastic, this loss of negative pressure would be increased. Also, as shown by Roy and Adami, ${ }^{8}$ a dilated heart does not thoroughly empty itself and this, again, would diminish its suction-power. So that if, as I have endeavoured to show, cases with early diastolic murmur have a dilated left ventricle the murmur cannot be explained by the negative intra-ventricular pressure, as this would be considerably less in such hearts than in those with a left ventricle of normal size. Another great objection to all these views is that they make no attempt to explain why the murmur should only occasionally be

2 Giuffré: Cinquième Congrès de la Société Italienne de Médecine, Dctobre, 1892 .

3 Brit. Med. Jour., vol. i., 1897, p. 200.
4 Text-book of Medicine, vol. ii., p. 74.

Diseases of the Heart, third edition, p. 132.

6 St. Bartholomew's Hospital Reports, 1888, p. 200

7 Diseases of the Heart, p 532. in the early part of the diastole, as such causes would be equally potent in all cases of stenosis. There is, lastly, the explanation put forward by Petit ${ }^{9}$ that the diastolic murmur is only heard when the heart is beating slowly, as then the ventricular systole being more complete the left ventricle is better emptied, and there is therefore a more energetic flow of blood into the ventricle at the beginning of diastole, producing a murmur immediately after the second sound. This connexion between the early diastolic murmur and a slow strongly-beating heart is, however, quite disproved, as mentioned before, by clinical examination, the murmur being rather heard with a failing heart.

For these reasons it seems to me that the suggested causes of this murmur in the early half of the diastole are insuffcient. If, as I suggest, this early diastolic murmur is always associated with free mitral regurgitation the explanation is simple. The ventricular systole drives a certain quantity of blood back through the constricted orifice into the already over-distended left auricle and pulmonary veins, and at the beginning of diastole these cavities by their elastic recoil drive some of the blood back through the narrowed mitral valve, thus producing the murmur.

This view of the dependence of the early diastolic murmur of mitral stenosis on co-existing regurgitation thus seems to be supported by some clinical and post-mortem evidence: it affords a simple solution of the mechanism of this rather obscure murmur and also explains why it is only heard in a certain number of cases of mitral stenosis.

Gower-street, W.C.

\section{THE ACTION OF THE WATER OF LLAN- GAMMARCH WELLS ON URIC ACID.}

BY W. BLACK JONES, M.D., B.S. LOND., D.P.H., RESIDENT PHYSICIAN, LLANGAMMARCH WELIS SPA; AND

EDWARD RUSSELL, B.Sc. LOND., F.C.S., F.I.C., SENIOR DEMONSTRATOR OF STATE MEDICNE, KING'S COLLEGE.

THE well-known efficacy of waters containing salts of the alkaline earths in the treatment of lithæmia has induced us to record the following observations on the water of Llangammarch Wells which contains the chlorides of barium and calcium in solution.

A man, aged 66 years, who had suffered for many years from symptoms of renal calculus, consulted one of us (Dr. W. Black Jones) in September, 1896. For the past 10 years he had been taking Contrexéville and Wildungen waters, but without relief. In 1895 he began taking the water of Llangammarch Wells, but in the following year was still suffering from the same symptoms. His urine was acid and the specific gravity was 1020. There was no albumin or sugar present, but he passed a large quantity of uric acid crystals. He suffered from obscure pains in the abdomen and had also well-marked signs of osteo-arthritis in the right knee and in the phalanges of the fingers. In May, 1897, he consulted Dr, Lauder Brunton who reported that all the symptoms of calculus had disappeared. Since then his urine has been free from deposit of uric acid, his rheumatism is much better, and his health is generally improved. He has continued to take a glass of Llangammarch Wells water daily.

The success of the above case induced one of us (Mr. Edward Russell) to make the following series of experiments upon himself. As a preliminary the alkalinity of the following waters was tested with a decinormal solution of hydrochloric acid, 20 cubic centimetres of the water being used in each case. It was found that distilled water was neutralised by 0.1 cubic centimetre of acid, tap water (New River) by 0.8 cubic centimetre, and the Llangammarch Wells water by 0.36 cubic centimetre. The direct action of the above waters on uric acid was then tested in the following manner. 250 cubic centimetres of the water with about one gramme of powdered uric acid were kept at $100^{\circ} \mathrm{F}$. in a bath for some hours ; 20 cubic centimetres of the solution were then taken, filtered, and treated with standard solution of permanganate of potassium. The results, which are denoted by the amount 
of solution of permanganate of potassium used, were as follows :-

$\begin{array}{lllllr}\text { Distilled water } & \ldots & \ldots & \ldots & \ldots & 2.4 \text { cubic centimetres. } \\ \text { Tap } & \end{array}$

Llangammarch Wells water ... 6.6 " "

The amount of uric acid voided in the urine was estimated as follows: 100 cubic centimetres of urine were mixed with 30 grammes of chloride of ammonium. This solution was allowed to stand for two hours, being frequently stirred, and was then filtered and washed with a saturated solution of sulphate of ammonium; the precipitate was washed off the filter-paper and after adding 20 cubic centimetres of sulphuric acid it was titrated with a standard solution of permanganate of potassium by Hopkins's method.

The experimenter at this time was suffering from obscure pains in the lumbar region and was passing rather less urine than normal, with a heavy deposit of urates. He was drinking about two and a half pints of liquid per diem, chiefly tea and New River water. $\mathrm{He}$ was taking a moderate meat diet. After a fortnight's dieting about two and a half pints of urine were passed in 24 hours the specific gravity of which was 1030 . The reaction was acid and on analysis there was found to be 0.058 gramme of uric acid per 100 cubic centimetres. A glass of the Llangammarch Wells water was then substituted for the New River water. This was taken twice daily before meals, very little other liquid being taken. This diet was continued for a fortnight. It was then found that three and a quarter pints of urine were passed in 24 hours, the specific gravity of which was 1022 , and 0.066 gramme of uric acid was passed per 100 cubic centimetres. After the mineral water had been discontinued for a fortnight two and a quarter pints were passed per diem, the uric acid amounting to 0.09 gramme per 100 cubic centimetres. The results are expressed in the following table.

TABLE $\mathrm{I}$.

\begin{tabular}{|c|c|c|c|c|}
\hline Diet. & $\begin{array}{l}\text { Amount of } \\
\text { urine in } \\
\text { cubic centi- } \\
\text { metres. }\end{array}$ & $\begin{array}{c}\text { Increase } \\
\text { per } \\
\text { cent. }\end{array}$ & $\begin{array}{l}\text { Amount of } \\
\text { uric acid } \\
\text { in grammes. }\end{array}$ & $\begin{array}{c}\text { Increase } \\
\text { per } \\
\text { cent. }\end{array}$ \\
\hline I. Normal ... ... & 1440 & - & 0.83 & - \\
\hline 2. Mineral water & 1900 & 31 & $1 \cdot 25$ & 50 \\
\hline 3. Normal ... ... & 1300 & - & $1 \cdot 18$ & 42 \\
\hline
\end{tabular}

In each of the above cases the results represent a mean of two analyses.

A second series of experiments was then conducted, the urine being analysed while a normal diet was being taken and again after a mineral water diet of six days' duration. The results were as follows :-

TABLE II.

\begin{tabular}{c|c|c|c|c}
\hline Diet. & $\begin{array}{c}\text { Amount of } \\
\text { urine in } \\
\text { cubic enti- } \\
\text { metres. }\end{array}$ & $\begin{array}{c}\text { Increase } \\
\text { per } \\
\text { cent. }\end{array}$ & $\begin{array}{c}\text { Amount of } \\
\text { urie acid } \\
\text { in grammes. }\end{array}$ & $\begin{array}{c}\text { Increase } \\
\text { per } \\
\text { cent. }\end{array}$ \\
\hline 1. Normal ... ... & 1130 & - & $1 \cdot 03$ & - \\
2. Mineral water & 1530 & 35 & $1 \cdot 34$ & 30 \\
\hline
\end{tabular}

We conclude from the above observations that the effect of substituting the Llangammarch Wells water for ordinary water is to increase the quantity of urine passed and also to increase the output of uric acid. This increase of the latter is also maintained to a certain extent when the water is no longer taken, but gradually returns to the normal for that individual.

The relation of uric acid to the various manifestations of gout and its deposition in the kidney in the form of calculus has been studied during recent years by several eminent members of the medical profession who have contributed much to our information on the subject. Considerable advances have been made in our knowledge as to its circulation in the blood and the factors determining its deposition in the tissues. The influences affecting its elimination from the body are, however, less well known to us. Many attempts have been made to discover a solvent of uric acid, but these experiments have not as yet been successful. Several mineral waters have long enjoyed a repute in the treatment of this diathesis, but most of them do not act as solvents at all, their action being chiefly diuretic. Some of the well-known waters would even seem to be contra-indicated on chemical grounds, although clinically the results are good. According to an analysis of Contrexéville water, for example, sulphate of calcium is the only mineral ingredient occurring in any important quantity in this water.

In the above experiments it will be noticed that chemically tap water is a better solvent of uric acid than Llangammarch Wells water, yet when the latter was substituted for the former clinically there was an increase of the output of from 30 to 50 per cent. It will be observed that the increased diuresis was due to the action of the mineral water alone, as the same quantity of liquids was taken in each case. The action of the Llangammarch Wells water in eliminating uric acid has thus an important bearing on the treatment of chronic gout and allied diseases. The following evidence gives the results of its employment in such cases. A number of patients visit Llangammarch annually suffering from gouty swellings of the joints. After a course of mineral water in nearly every case there is considerable benefit, the swellings decrease, there is less pain, and there is less stiffness in the joint. In many cases baths of the mineral water and also massage are used with very good results. In a few instances which at first appeared most unpromising considerable relief has been obtained. In the same manner there has been success in the treatment of osteo-arthritis. We therefore consider that in the Llangammarch Wells water we have a valuable adjunct to the present treatment of the uric acid diathesis, that substance being eliminated in considerable amount, hence this water is indicated in cases of gout, osteo-arthritis, uric acid, gravel, and calculus. In addition to the mineral water treatment there are at Llangammarch the advantages of a spa within a few hours' journey from London, together with a mountain atmosphere and such various forms of exercise as walking, hill-climbing, golf, \&c., which are invaluable aids to the balneologist.

\section{M} TEDICAL, SURGICAL, OBSTETRICAL, AND THERAPEUTICAL.

\section{A NOTE ON DIAPHRAGMATIC PLEURISY.}

By George A. Clarkson, F.R.C.S. Eng.

THE subject of diaphragmatic pleurisy is of exceptional interest to the surgeon on account of the symptoms being so very suggestive of some grave abdominal affection. The differential diagnosis is generally far from easy, for few signs, if any, are discoverable on the most carcful examination and in the majority of cases no effusion follows. The most striking symptom is severe pain in the upper part of the abdomen. The diaphragm is fixed and the abdominal walls consequently do not move on respiration. The respiration is quickened and expansion is confined to the upper part of the thorax. There is generally some accompanying fever. Coupled with these symptoms is one of the most marked features in the disease, an extremely anxions countenance. The expression is such as one is accustomed to see in bad abdominal lesions. Some cases of pneumonia present this train of symptoms at the onset and I suppose that there can be no doubt that this is due to the presence of diaphragmatic pleurisy. The following case which came under my care illustrates in a striking manner most of these points. Late one evening I was asked to see a boy, aged 11 years, whose mother gave me the following history. He had been perfectly well in the middle of the day when he came home to dinner and partook of a good meal. He then went out to play with some other boys and returned home ill and evidently in great pain. When I examined him there were no bruises or any signs whatever of injury on the body and no broken ribs. He complained of acute pain in the abdomen and lay on his back with his knees drawn up. The abdomen was somewhat distended, motionless, and very rigid. It was tender on 